\title{
An Investigation into the Modelling Challenges for Overland Flow Path Mapping and the Analysis of Practical Solutions
}

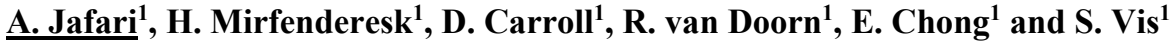 \\ ${ }^{1}$ Natural Hazards Team, City Planning, City of Gold Coast
}

Email: ajafari@goldcoast.qld.gov.au

\begin{abstract}
The lengthy computation time and very high computing capacity required to simulate high resolution catchment-wide stormwater models are significant challenges in developing overland flow path maps. Current practise tends to sub-divide a catchment into many sub-catchments, each with own stormwater pipe network in order to reduce the computing capacity, however this method tends to promote further complications due to cross-flows and backwater effects, particularly in flat catchments. An alternative approach is to remove the stormwater network and to estimate its impact through loss parameters. The first approach resolves the computing capacity limitations; however, the required modelling time remains an issue. In addition, full implementation of this approach requires an estimation of sheet flows and tailwater conditions at the boundary of each sub-catchment which can be very difficult to implement in relatively flat catchments. The second approach resolves both boundary condition and run time issues. This investigation involved a case study to examine the feasibility as well as the pros and cons of both approaches. Currumbin Creek, located in the southern part of the Gold Coast, was selected as a case study. First a coupled 1D-2D model of the catchment was developed to establish the most accurate overland flow path. Then two sets of overland flow paths were developed using the two abovementioned approximate approaches. Their results were compared with the results of the whole of catchment model to establish the better approach. The study shows that the second approach provides better results. It is important to note that the accuracy of the second approach depends on assigning an appropriate loss parameter to account for drainage capacity of the stormwater networks. In this study, various conceptual hydrological models were proposed to assess loss parameter estimation by finding a relationship between the volume of water conveyed by the stormwater pipe network and the input rainfall hyetograph. The study contributes to the body of knowledge by providing further insight into overland flow path modelling and mapping.
\end{abstract}

Keywords: Overland flow path, flood modelling, hydrology, rainfall hyetograph, stormwater modelling 
Jafari et al., An investigation into the modelling challenges for overland flow path mapping and the analysis of practical solutions

\section{INTRODUCTION}

Overland flow is generally shallow fast moving stormwater often carrying debris during intense rainfall events that can pose a significant danger to the community. It is crucial for both city planners and emergency management personnel to have a high resolution city-wide map of the City's overland flow paths (OLFPs), so that they have the ability to identify potential hotspots. The computation time required using coupled Central Processing Unit (CPU) based hydrodynamic models is the main obstacle in providing such a map. This obstacle can be overcome partly by using hydrodynamic models that employ Graphic Processing Unit (GPU) solvers. However, GPU solvers are not yet enabled to undertake 1D-2D coupled modelling. To overcome this shortcoming, OLFP modelling was undertaken using a high resolution two-dimensional modelling scheme by taking into account appropriate volume losses for the underground 1D pipe network in urbanized areas. The main challenge in this study was to find a relationship between the volume of water discharged by the stormwater pipe network and an input rainfall hyetograph for a 1 in 100 year event. In this way the input hyetographs can be adjusted within a 2D model in such a way that the results are similar to those that included the stormwater network system.

Various hydrological abstraction schemes were investigated in this study and the results compared to a 1D-2D coupled model for the same catchment. The Currumbin Creek Catchment, located in the southern part of the Gold Coast, was selected for the purposes of this investigation. The coupled stormwater model was developed for the downstream part of the catchment which is highly urbanized. The maximum depth, water elevation and flow rates were then extracted for different locations to evaluate and compare each of the applied hydrological abstraction methods.

The adapted methodology used the TUFLOW hydrodynamic with applied direct rainfall using the latest available LIDAR data (2009 and 2012). The hydrological inputs were obtained from the Currumbin URBS model [1]. This model is divided into 21 sub-catchments. Rainfall was varied spatially by applying uniform rainfall of a given duration over each sub-catchment to which the appropriate regional design temporal pattern was applied. A constant Mean High Water Springs (MHWS) tailwater level of $0.66 \mathrm{~m}$ AHD was assumed.

\section{STUDY AREA}

This investigation was conducted through a case study of the Currumbin Creek Catchment which is located in southern part of Gold Coast City and covers an area approximately $51.7 \mathrm{~km}^{2}$. The catchment extends from the South-West to the North-East. It is bounded by Mount Cougal and Mount Tomewin to the west, Piggabeen and Cobaki Creek catchments (NSW) to the south, Tallebudgera Creek catchment to the north and the Pacific Ocean to the east. The upper reaches of the creek are quite steep with narrow channels and densely forested areas. The middle reaches are flatter but remain forested. The lower reaches are heavily urbanised and have flat floodplains with some remnants of native vegetation in riparian areas. There are no significant storages (dams) in the catchment. Figure 1 shows the locality map of the Currumbin Creek catchment.

\section{METHODOLOGY}

A one-metre grid Digital Elevation Model (DEM) was developed and used to represent the Currumbin OLFP model topography. The catchment was modelled by TUFLOW hydrodynamic model [2]. This software is capable of simulating complex floodplains and canal systems using hydro-dynamically coupled two-dimensional topographic grids with a "rain on grid" approach. Three alternative

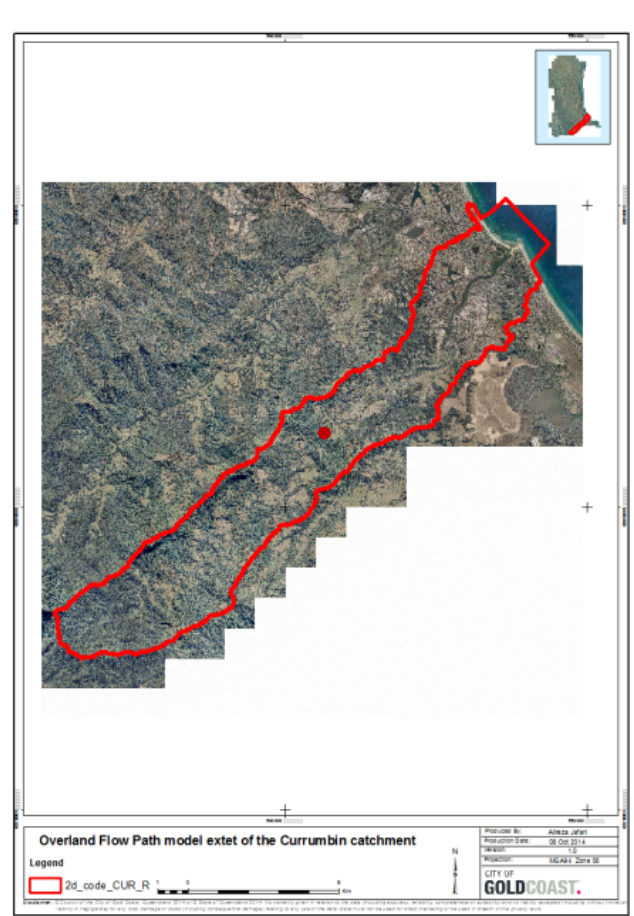

Figure 1. Currumbin OLFP model Extent. simulation methods were investigated in this study; (i) Detailed 1D2D model of the whole catchment, including all the stormwater network, (ii) Detailed 1D-2D sub-divided catchment models of the study area including all stormwater networks, (iii) Detailed 2D hydrodynamic model of the whole catchment, with the stormwater network approximated through hydrological loss parameters. A comparison between these alternatives will show the most effective methodology and also provide a measure of accuracy of the selected loss parameters used in alternative (iii). A $4 \mathrm{~m}$ grid model with one second time-step was used as a base configuration for each method. 
Jafari et al., An investigation into the modelling challenges for overland flow path mapping and the analysis of practical solutions

\section{MODELLING AND DISCUSSION}

The TUFLOW GPU solver module was used to simulate the rain on grid process in alternative (iii) and the CPU solver module was used to simulate the rain on grid stormwater network coupled with 2D model in alternatives (i) and (ii). The hydrologic inputs to the models were based on URBS hydrological model [3]. The URBS model was calibrated to the January 2008 and June 2005 flood events. Figure 2 shows the URBS model calibration at the Nichols Bridge ALERT station for the June 2005 event $\left(R^{2}=0.91\right)[1]$. The location of the Nichols Bridge ALERT station is shown in Figure 1 as a red dot. For the purpose of this investigation, the 1 in 100 ARI 1 hour duration $(\mathrm{Q} 100 \mathrm{H} 1)$ design storm event was used as the representative storm event for this study. The AWE2000 temporal patterns derived specifically for the Gold Coast area were applied to this design event.

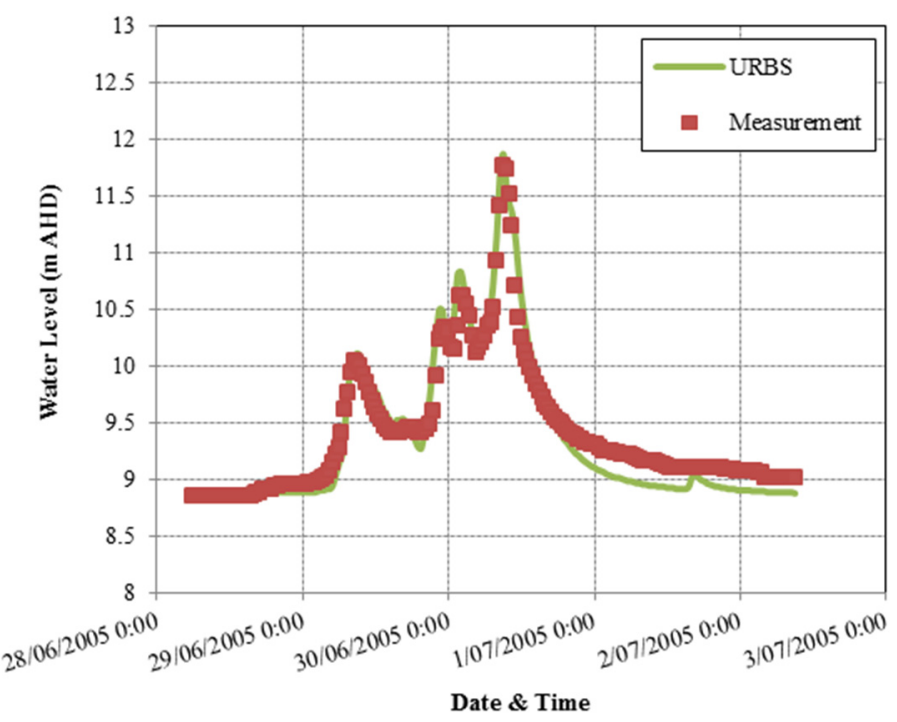

Figure 2. Hydrology model calibration at Nichols Bridge.

\subsection{Incorporate entire stormwater network in the catchment (1D-2D coupled model)}

Technically, the most ideal model to simulate overland flow path behaviour is one that models the entire stormwater pipe network [6]. This involves employing 1D-2D coupled models that use CPU solvers. For this case study, a stormwater pipe network model was developed for the entire Currumbin Catchment. The input design rainfall was based on a 1 in 100 year ARI (Q100) storm of 1 hour (H1) duration. A tailwater level of $0.66 \mathrm{~m}$ (MHWS) was assigned as the downstream boundary condition. The maximum water surface level map (MWSL) for this event was then computed and is shown in Figure 3-A. Modelling was undertaken using a purpose built high end CPU computer noting that such modelling for large catchments is currently impractical. For ease of referencing, this approach is named the Entire Stormwater Network (ESN) model.

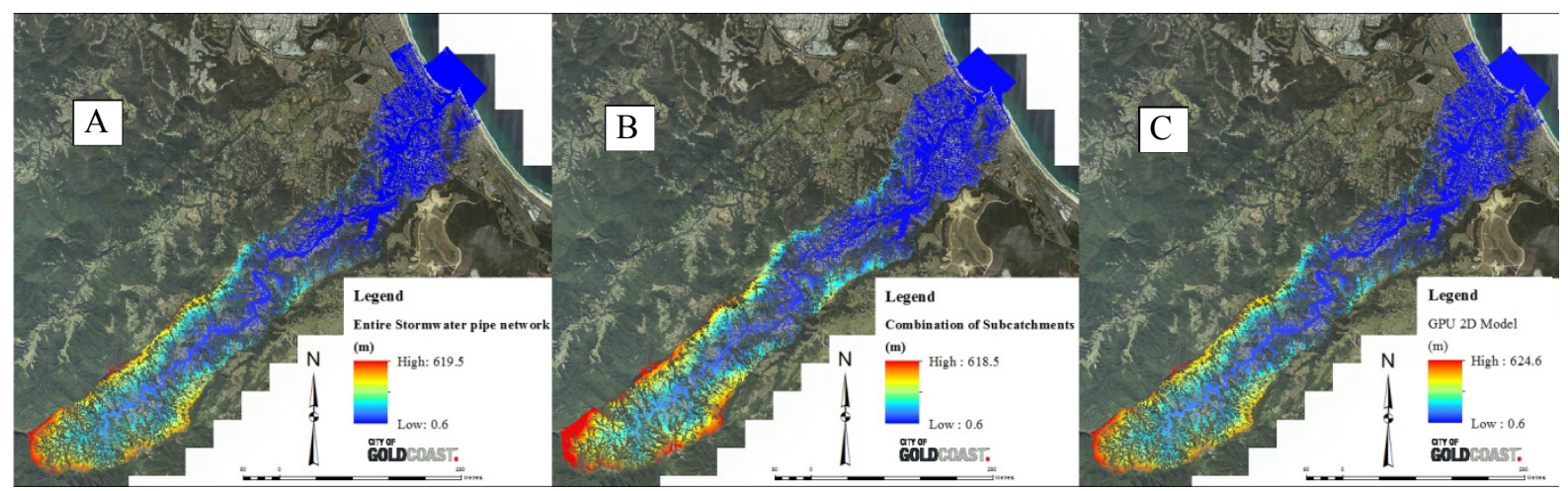

Figure 3. Maximum water level output of different modelling approaches. A: Entire stormwater pipe network model result, B: Combination of individual subcatchments, C: 2D model with no stormwater pipe

\subsection{Combination of individual sub-catchments (1D-2D coupled model)}

This is an alternative to the ESN approach in that it incorporates the stormwater network but based on modelling individual catchments to reduce computation times, nevertheless computing times are still lengthy as numerous sub-catchments are required to accurately model the whole catchment. The main issues with this method are (a) the cross flows between sub-catchments and (b) setting appropriate downstream tailwater levels. Further, modelling has to proceed from downstream to upstream to facilitate tailwater level setting. Upstream inflows are sourced from the hydrology model. Hence, the accuracy of the results is highly influenced by the accuracy of hydrology model, especially for downstream sub-catchments which in this case study are heavily urbanized. Similar to the ESN model a Q100H1 event with a MHWS tailwater level was adopted for this approach. The 
Jafari et al., An investigation into the modelling challenges for overland flow path mapping and the analysis of practical solutions

MWSL output of all sub-catchments were combined and the results are shown in Figure 3-B. For ease of referencing this approach is named as the Combination of Individual Sub-catchments (CIS) model.

\subsection{Two dimensional (2D) GPU model}

The computation time required using coupled CPU based hydrodynamic models is the main obstacle in preparing OLFP maps. This obstacle can be overcome by using hydrodynamic models that employ GPU solvers as they are up to 100 times faster than CPU solvers [4].

Assumed roughness values for hydraulic structures such as bridges and culverts were adjusted in order to replicate hydraulic afflux effects. The model was run for the same event scenario (Q100H1) and the model results of maximum water level surface (MWSL) is shown in Figure 3-C. For ease of referencing this approach is referred as the Two Dimensional GPU (2DGPU) model. Table 1 shows the run time of each of the aforementioned models based on their computational log. Apparently the 2DGPU approach is 7.5 and 13 times faster than CIS and ESN approaches respectively.

Table 1. Computational time of each model.

\begin{tabular}{|llll|}
\hline Model & $\begin{array}{l}\text { Entire Stormwater } \\
\text { Network (ESN) }\end{array}$ & $\begin{array}{l}\text { Combination of Individual } \\
\text { Sub-catchments (CIS) }\end{array}$ & $\begin{array}{l}\text { Two Dimensional } \\
\text { GPU (2DGPU) }\end{array}$ \\
\hline Log time (hh:mm:ss) & $22: 06: 10$ & $12: 44: 20$ & $1: 42: 10$ \\
\hline
\end{tabular}

\subsection{Comparison CIS \& 2DGPU}

The ESN model was used as the basis to assess the performance of the other approaches. Differences for both the CIS and the 2DGPU maximum water surface levels and the benchmark ESN model were calculated for each computational grid cell. These differences are shown in Figure 4.

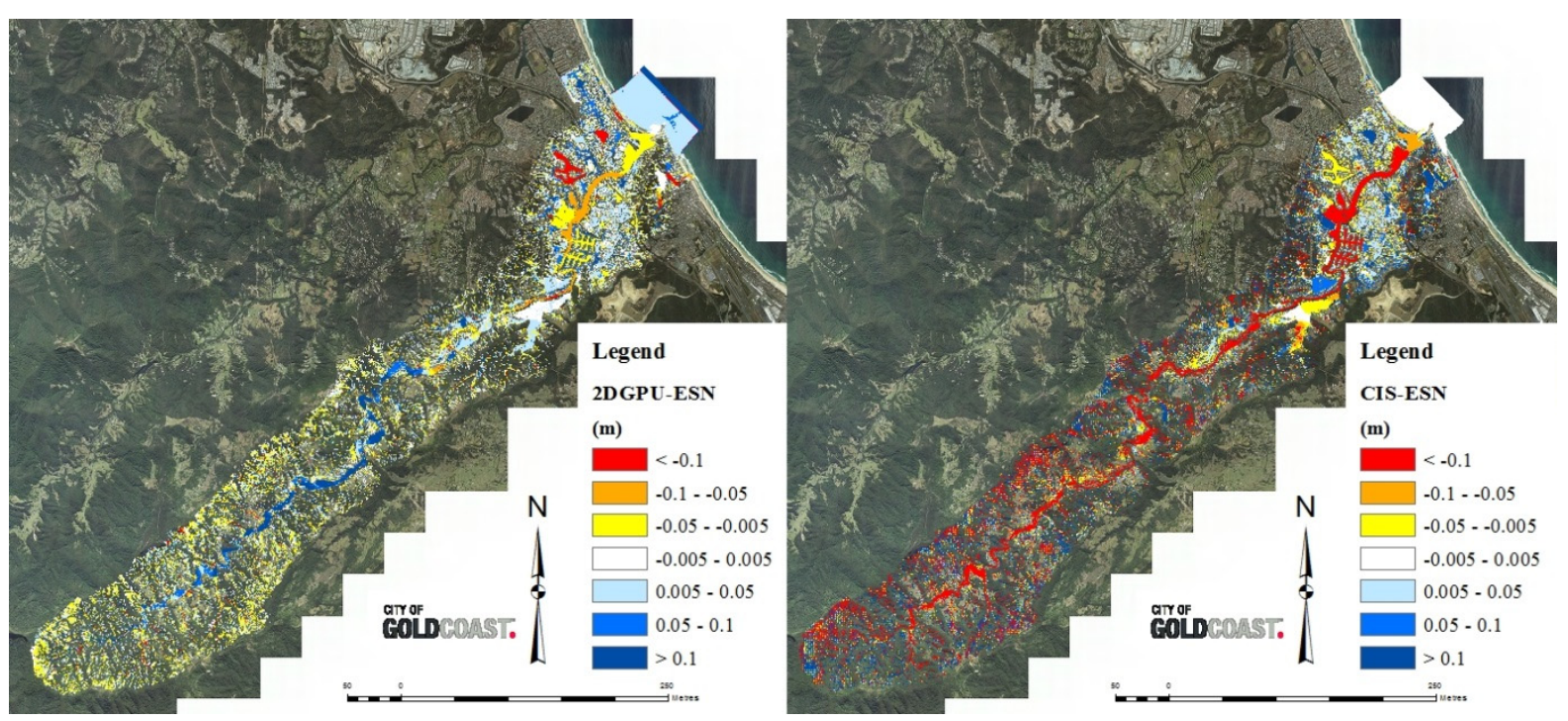

Figure 4. Maximum water surface level differences between 2DGPU \&ENS (left) and CIS \& ESN (right)

In Figure 4, the 2DGPU model tends to predict higher MWSL's in the upper reaches of the main stream of the Currumbin Creek and consequently higher MWSL's in the middle reaches. Interestingly in the downstream areas of the catchment that are highly urbanized, the order of differences between 2DGPU approach and ENS is generally between $0.005 \mathrm{~m}$ to $0.05 \mathrm{~m}$. This is because the downstream of the catchment is relatively flat and the tailwater level, which is set to MHWS, filled up the most of

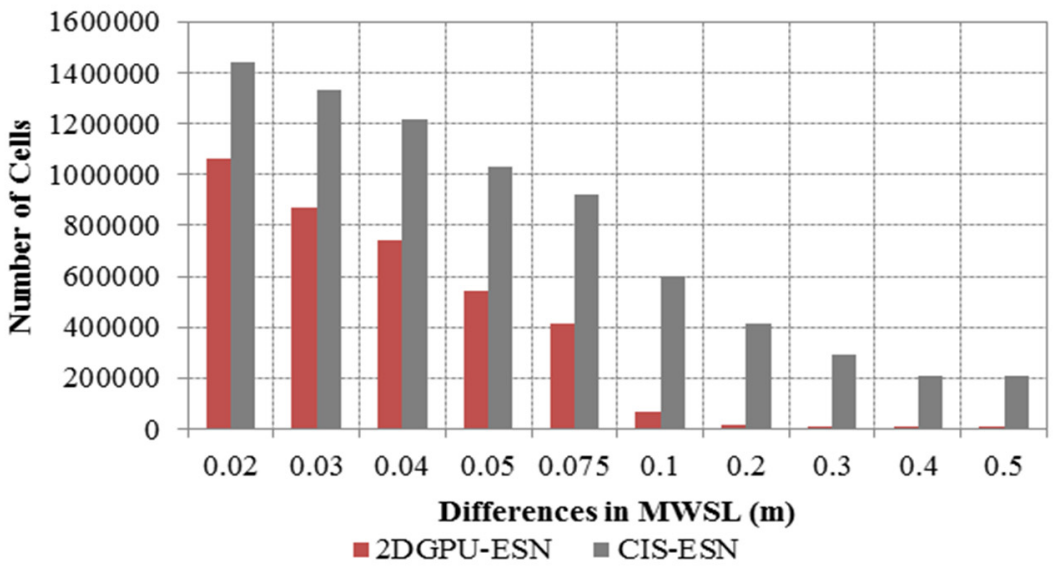

Figure 5. Histogram of absolute difference between 2DGPU and CIS with ESN. 
Jafari et al., An investigation into the modelling challenges for overland flow path mapping and the analysis of practical solutions

the lower reach pipes and consequently compromised their capacities.

On the other hand, a relatively uniform difference pattern can be seen in the difference map for CIS and ESN. Generally in the main stream of the Currumbin Creek, differences are over $0.1 \mathrm{~m}$ (shown coloured in red on Figure 4). Basically the CIS approach tends to predict lower MWSL's than the ESN throughout the whole catchment. The main reason for this is due to the differences in the estimated upstream sub-catchment inflows and downstream tailwater levels between the CIS and ESN models. These differences tend to propagate downstream. A histogram of absolute differences in MWSL values is plotted in Figure 5. As can be seen, the 2DGPU approach provides a better prediction in terms of both qualitative (c.f. Figure 4) and quantitative (c.f. Figure 5) assessment compared to the CIS approach when benchmarked against the ESN results. Accordingly, in the absence of inadequate computational power to run an ESN model, the 2DGPU is considered to be a better approach taking into account its inherent simplicity compared to the uncertainties associated with CIS approach.

\subsection{Adopting various hydrological techniques to consider pipe network effect}

The GPU solver is not yet enabled to undertake 1D-2D coupled modelling. Therefore, an OLFP study needs to be done using a high resolution two-dimensional modelling scheme by taking into account appropriate volume losses for the underground 1D pipe network in urbanized areas. To use this method there is a need to find a relationship between the volume of water discharged by the stormwater pipe network and an input rainfall hyetograph for a 1 in 100 year ARI 1 hour event. In this way the input hyetographs can be adjusted for a 2D model in such a way that the results are similar to those that included the stormwater network system. Various hydrological abstraction schemes were investigated in this study and the results compared to a 1D-2D coupled model for the same catchment:

\section{- No losses applied to rainfall hyetograph}

This model was developed assuming that the stormwater pipe network has no contribution to discharging stormwater to the creek or ocean. Basically it is assumed that the pipe network capacity is minimal due to downstream tide levels. Moreover, this model was used as a benchmark to assess the various hydrological abstraction schemes that simulate the stormwater network capacity. The adopted storm event for this scenario was again the Q100H1 event. A 1 in 2 year ARI event (Q2) was assumed to be the equivalent of an average capacity of a stormwater pipe network in the Currumbin Catchment. For modelling purposes it was assumed that the pipe network has the capacity of draining a Q2 ARI event of 1 hour duration. It should be noted that the choice of a 1 in 2 year ARI event to represent the stormwater network capacity is based on typical stormwater drainage design standards and merits further discussion however such discussion is beyond the scope of this study.

\section{- $\quad$ Rain on sub-catchment Area, Classic method (ROSAO)}

In this method the Q2 was deducted from input rainfall hyetograph $(\mathrm{Q} 100)$ for each time step of the temporal pattern for those sub-catchment areas where the stormwater pipe network infrastructure exists. In the upper reaches of the catchment, where there is no urbanization, no such deduction was made (see Figure 6).

\section{- Rain on Sub-catchment Area, Alternative Method 1 (ROSA1)}

This approach was similar to ROSA0 method, however for each sub-catchment the Q2 rainfall was scaled according to the degree of urbanization for that sub-catchment. The degree of urbanization is measured as the fraction of the sub-catchment area that is urbanized (U) [5]. Basically if the sub-catchment is fully urbanized, the input hyetograph of ROSA1 and the ROSA0 method would be the same (see Figure 6).

\section{- $\quad$ Rain on Sub-catchment Area, Alternative Method 2(ROSA2)}

This approach assumes that the capacity of the pipe network is constant throughout the rainfall event, via application of a uniform temporal pattern to the Q2 event. Adopting this approach requires deduction of an averaged Q2 rainfall per time interval from the Q100H1 hyetograph. Also the factor of the sub-catchment area that is urbanized was included as per the ROSA1 method (see Figure 6).

\section{- Rain on Sub-catchment Area, Alternative Method 3(ROSA3)}

In this method the input rainfall hyetograph was generated using the "Chicago Storm Method" [6] which is to build a hyetograph starting at the centre such that the ARI for each sub-duration is the same as that for the entire event. Then the averaged Q2 (as per ROSA2) was modified according to the degree of urbanization (as per ROSA1) is deducted from the input hyetograph (see Figure 6).

All the above mentioned methods were used in the 2DGPU approach and results were compared to the ESN model. The error histogram shown in Figure 7 indicates that the ROSA1 methodology is better than the other methods in providing the closest results to ESN in terms of maximum water depths. 
Jafari et al., An investigation into the modelling challenges for overland flow path mapping and the analysis of practical solutions

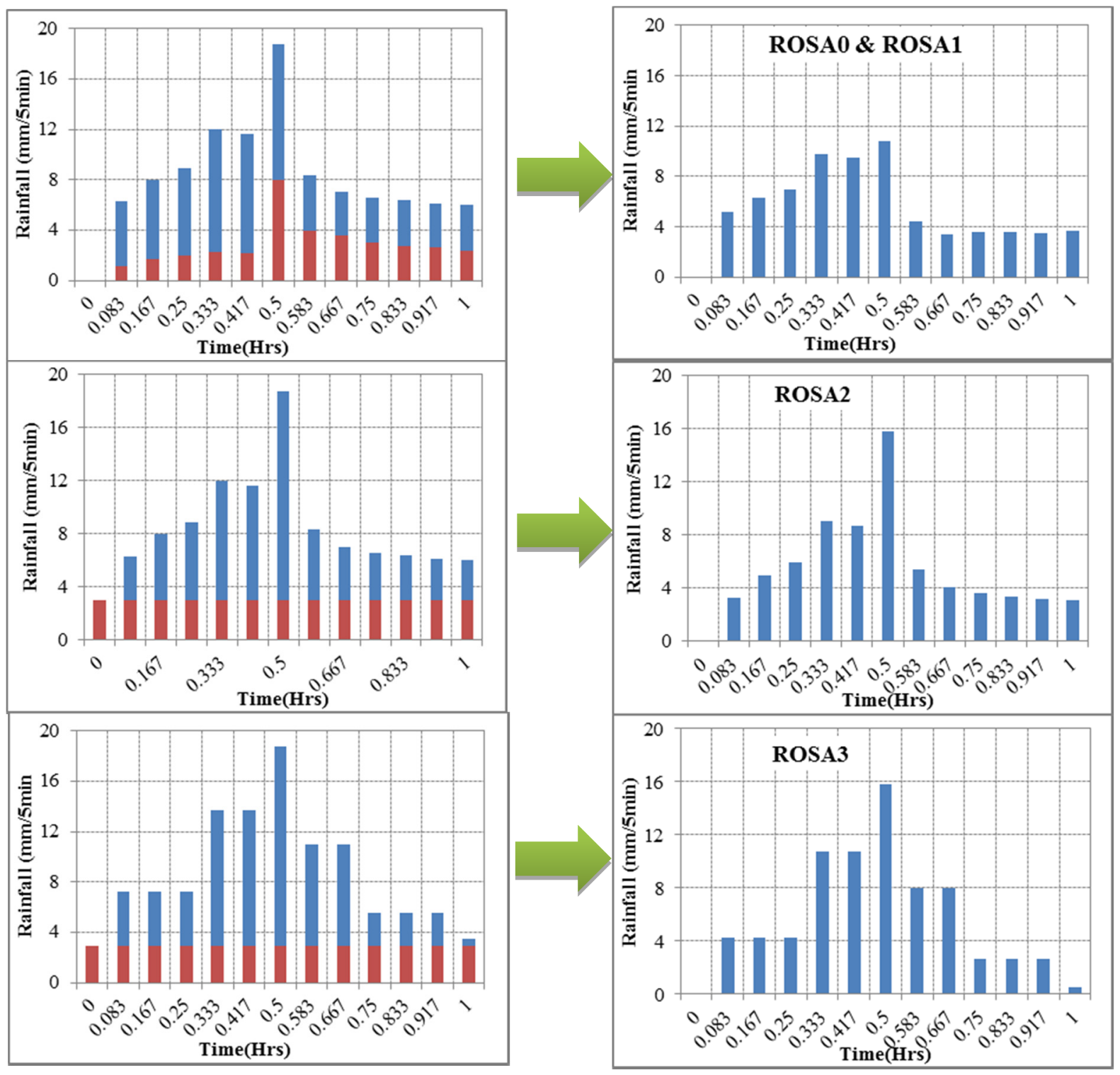

Figure 6. Rainfall losses (Q2H1) were applied to the input rainfall hyetograph $(\mathrm{Q} 100 \mathrm{H} 1)$ for downstream subcatchments of Currumbin Catchment. Blue bar: Q100H1, Red bar: Q2H1 (constant red bar is an average hyetograph).

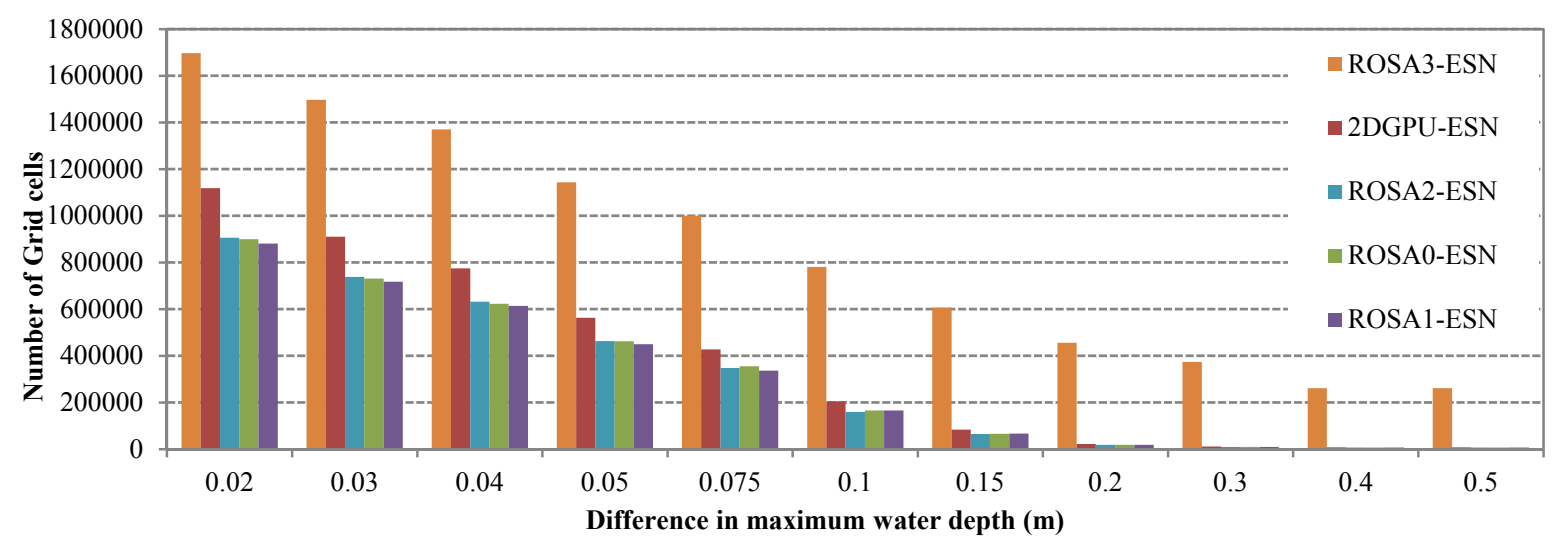

Figure 7. Histogram of absolute difference between 2DGPU, ROSA0, ROSA1, ROSA2, and ROSA3 with ESN.

ROSA0 and ROSA2 provided comparable results but not as good as ROSA1. ROSA0 has the disadvantage that it requires discretion as to which sub-catchments a deduction is made for the pipe system, whereas ROSA1 
Jafari et al., An investigation into the modelling challenges for overland flow path mapping and the analysis of practical solutions

applies an urbanization index that can be readily calculated using GIS. ROSA2 has the advantage that it does not require an assumed temporal pattern for the Q2 event. All three approaches (ROSA0, ROSA1 and ROSA2) are markedly better than the 2DGPU results when compared to the ESN results. It is concluded therefore that some allowance should be made for the underground pipe system. The extent of this allowance is a function of the efficiency of the existing stormwater network and would require calibration when applied to a particular catchment.

ROSA3 on the other hand shows poor agreement with the ESN results. It should be noted that the ROSA3 uses the "Chicago storm design method" which results is a much peakier temporal pattern than the AWE2000 temporal pattern used in this study. It would have been better to compare this approach to ESN results that are also based on the "Chicago storm" pattern. Nevertheless, the methodology is attractive in that it does not require application of a "design" temporal pattern and all sub-durations are critically accounted for. Application of a constant deduction of the pipe system also assists in the generality of this approach. The results of this approach are considered to be conservative but nevertheless the methodology does merit further investigation because of its generality.

\section{CONCLUSION}

It is very important to have a high resolution city-wide map of the City's overland flow paths (OLFPs) for development and planning purposes. A fully hydrodynamic coupled 1D-2D simulation of large catchments using CPU solvers is not practical due to inadequate computer processing power. GPU solver technology is promising, but it is not yet capable of coupled 1D-2D simulations. There are two other alternatives in resolving this shortcoming. One alternative is to divide the catchment into smaller sub-catchments and run a coupled 1D2D CPU model over each sub-catchment and when all completed, combine the sub-catchment maps as a single overland flow path map (CIS approach). The other option is to use a high resolution two-dimensional model with a GPU solver (2DGPU approach) and represent 1D elements through hydraulic and hydrologic parameterization. Both approaches were examined for a case study of the Currumbin Catchment Creek and results were compared to the benchmark 1D-2D coupled CPU ESN model. The comparison indicated that for this catchment the 2DGPU approach provides better results. Also considering the simplicity and computational speed of the 2DGPU approach against uncertainties and complexities associated with the CIS approach, further promotes the use of the 2DGPU modelling approach.

Additionally, as the 2DGPU model has not yet the capability to include the stormwater network, it was considered that modification of the input hyetographs (through application of hydrological losses) to account for the stormwater network would provide even better results. Various schemes were investigated in this study and the results compared to the 1D-2D coupled model for the Currumbin Creek Catchment ESN model. The results indicate that the ROSA1 model with time interval based scaled deduction (based on degree of urbanization) of a Q2 rainfall hyetograph provided the closest results in terms of maximum water depth. Therefore it can be concluded that for Currumbin Creek catchment the ROSA1 approach is the best available alternative to the 1D2D coupled model in undertaking OLFP modelling.

Finally, it should be noted that the lower reaches of Currumbin Creek are tidal and as such the capacity of the stormwater network is limited during large rainfall events with relatively high downstream tide levels (MHWS) as applied in this comparative analysis. Therefore caution should be exercised in extrapolating these results to other catchments. It is planned to undertake further comparative analysis for non-tidal urbanized catchments and these results will be reported in future papers.

\section{REFERENCES}

1. Gold Coast City Council (2013). Currumbin Creek Catchment Hydrological Study. Currumbin Creek Catchment Hydrological Study

2. BMT WBM (2010), TUFLOW 1D/2D hydrodynamic computational engine, User Manual2010-10-AB

3. Carroll D. G. (2012) Manual of Unified River Basin Simulator (URBS), A Rainfall Runoff Routing Model for Flood Forecasting \& Design

4. Syme B., Collecutt G., Ryan. P. (2012), Rapid and Accurate Flood Modelling Fully 2D GPU Solver, FMA Conference, Sacramento, USA2012

5. Carroll D.G. (1995), Assessment of the Effects of Urbanisation, Proceedings, 2nd Intl Stormwater Management Conference, IE Aust, Melbourne July 1995.

6. Keifer, C. J., and Chu, H. H. (1957), Synthetic Storm Pattern for Drainage Design, Journal of Hydraulic Engineering (ASCE), 83 (HY4): 1-25. 